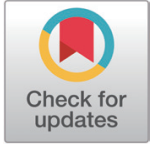

Received: Feb 10, 2021

Revised: Mar 3, 2021

Accepted: Mar 10, 2021

*Corresponding author

Sungkwon Park

Department of Food Science and

Biotechnology, Sejong University, Seoul

05006, Korea.

Tel: +82-2-3408-2906

E-mail: sungkwonpark@sejong.ac.kr

Copyright $@ 2021$ Korean Society of Animal Sciences and Technology.

This is an Open Access article distributed under the terms of the Creative Commons Attribution

Non-Commercial License (http:// creativecommons.org/licenses/by$\mathrm{nc} / 4.0 /$ ) which permits unrestricted non-commercial use, distribution, and reproduction in any medium, provided the original work is properly cited.

ORCID

Neeraja Recharla

https://orcid.org/0000-0001-9343-7436

Balamuralikrishnan Balasubramaniana

https://orcid.org/0000-0001-6938-1495

Minho Song

https://orcid.org/0000-0002-4515-5212

Pradeep Puligundla

https://orcid.org/0000-0002-2457-0273

Soo-ki Kim

https://orcid.org/0000-0003-3499-3330

Jin Young Jeong

https://orcid.org/0000-0002-8670-7036

\section{Dietary turmeric (Curcuma longa L.) supplementation improves growth performance, short-chain fatty acid production, and modulates bacterial composition of weaned piglets}

\author{
Neeraja Recharla ${ }^{1}$, Balamuralikrishnan Balasubramanian ${ }^{1}$, Minho Song ${ }^{2}$, \\ Pradeep Puligundla ${ }^{3}$, Soo-ki Kim ${ }^{4}$, Jin Young Jeong ${ }^{5}$ and Sungkwon Park ${ }^{1 *}$ \\ ${ }^{1}$ Department of Food Science and Biotechnology, Sejong University, Seoul 05006, Korea \\ ${ }^{2}$ Division of Animal and Dairy Science, Chungnam National University, Daejeon 34134, Korea \\ ${ }^{3}$ Department of Food Science and Biotechnology, Gachon University, Seongnam 13120, Korea \\ ${ }^{4}$ Department of Animal Science and Technology, Konkuk University, Seoul 05029, Korea \\ ${ }^{5}$ National Institute of Animal Science, RDA, Wanju 55365, Korea
}

\section{Abstract}

In livestock nutrition, natural feed additives are gaining increased attention as alternatives to antibiotic growth promoters to improve animal performance. This study investigated the effects of dietary turmeric supplementation on the growth performance and gut health of weaned piglets. A total of 48 weaned piglets (Duroc $\times$ [Landrace $\times$ Yorkshire]) were used in a 6-week feeding trial. All piglets were allotted to two dietary treatments: corn-soybean meal basal diet without turmeric (control) and with $1 \%$ weight per weight (w/w) turmeric powder (turmeric). The results showed that dietary inclusion of turmeric with the basal diet improved final body weight and total average daily gain $(p<0.05)$. The concentrations of short-chain fatty acids in the fecal samples, including acetic, butyric, and propionic acids, were higher in the turmeric group $(p<0.05)$. The villus height-to-crypt depth ratio was higher in the ileum of turmeric-fed piglets $(p=0.04)$. The $16 \mathrm{~S}$ rRNA gene sequencing of fecal microbiota indicated that, at the phylum level, Firmicutes and Bacteroidetes were the most predominant taxa in all fecal samples. Bacteroidetes were significantly decreased in the turmeric group compared to the control group $(p=0.021)$. At the genus level, turmeric showed a decreased abundance of Prevotella ( $p=0.021)$ and an increasing trend of Lactobacillus ( $p=0.083)$. Among the total detected species, nine bacterial species showed significant differences between the two groups. The results of this study indicated that turmeric altered the gut microbiota and shortchain fatty acid production. This suggests that turmeric could be used as a potential alternative growth promoter for piglets.

Keywords: Weaned piglets, Turmeric, Gut health, Gut microbiota, Growth promoters, 16S rRNA sequencing 
Sungkwon Park

https://orcid.org/0000-0002-7684-9719

\section{Competing interests}

Not applicable.

Funding sources

This work was carried out with the support of "Cooperative Research Program for Agriculture Science \& Technology Development (Project No. PJ015002032020),"Rural Development Administration and Basic Science Research Program through the National Research Foundation of Korea (NRF) funded by the Ministry of Science, ICT \& Future planning (2019R1F1A1059043), Korea.

Acknowledgements

Not applicable.

Availability of data and material Upon reasonable request, the datasets of this study can be available from the corresponding author.

Authors' contributions conceptualization: Recharla N, Park S. Data curation: Recharla N, Balasubramanian B. Formal analysis: Recharla N, Jeong JY. methodology: Kim S, Jeong JY. validation: Song M, Puligundla P. writing-original draft: Recharla $\mathrm{N}$ writing-review \& editing: Song M, Kim S, Jeong JY, Park S.

Ethics approval and consent to participate Animal care procedures and experimental protocols were approved by the Animal Care and Use Committee of Chungnam National University (Approval\# CNU-00611).

\section{INTRODUCTION}

Antibiotics have been widely used as growth promoters in livestock to improve animal performance and profitability by improving feed efficiency and animal growth, as well as reducing the incidence of diseases [1]. In particular, nursery pigs are supplemented with antibiotics for disease prevention or to reduce morbidity and mortality [2]. On the other hand, extensive use of antibiotics promotes antibiotic resistance, which could have a negative impact on both animal and human health. Antibiotics used for animal growth are closely related to the class of antibiotics used in human medicine to treat foodborne infections, including penicillin, aminoglycosides, and tetracyclines [3]. The use of antimicrobials in food-producing animals leads to multidrug resistance in both animals and humans $[3,4]$. Therefore, several countries have banned or placed restrictions on antibiotic use in animal feed [4-7]. Thus, it is necessary to adopt alternative approaches for antibiotic use. During post-weaning, various changes take place in the swine due to stress, new diet, and other factors, which cause increased invasion and colonization of pathogenic bacteria, resulting in infection and diarrhea $[8,9]$. The immune system is not mature enough in piglets to fight invading pathogens; hence, the post-weaning period is a critical time for maintaining animal health and performance. The gut microbiome plays a major role in immune system development, maintaining nutrient metabolism, performance, disease defense, and health status of the host [10]. Intestinal microbiota might be a potential novel strategy to modulate the general immune system and gut health $[11,12]$. To modulate the gut microbiota to exert beneficial effects on the host, most researchers have employed prebiotics, probiotics, essential oils [13-15], dietary enzymes, natural herbs, and medicinal plants [16] or phytobiotics [17-19].

Turmeric, also known as the golden spice, is a popular medicinal herb derived from Curcuma longa Linnaeus rhizomes. Turmeric plays a vital role in traditional medicinal purposesas an antimicrobial and anti-carcinogenic agent [20,21]. It contains approximately $69.4 \%$ carbohydrates, $5.1 \%$ fat, $6.3 \%$ protein, $3.5 \%$ minerals, and $13.1 \%$ moisture $[22,23]$. The turmeric rhizome contains a major fraction of starch $(47 \%-56 \% \mathrm{w} / \mathrm{w})$ on a dry basis $[24,25]$. Isolated turmeric starch contains $48 \%-50 \%(\mathrm{w} / \mathrm{w})$ amylose [25]. Indigestible carbohydrates, such as resistant starch and other carbohydrates, are fermented by microbes in the large intestine and produce short-chain fatty acids (SCFAs) and other products. The main bioactive compounds in turmeric are curcuminoids, which constitute $1 \%-6 \%$ of the dry weight of turmeric [26]. The three major curcuminoids are curcumin (80\%), desmethoxycurcumin (18\%), and bisdemethoxycurcumin $(2 \%)[27,28]$. The bioactive compounds of turmeric consist of volatile and non-volatile phytochemicals that are less toxic and have beneficial effects, including antioxidant, antibacterial, anti-inflammatory, antiviral, antifungal, anticarcinogenic, and hypo-cholesteric activities [29-31].

Turmeric has gained attention in recent years as a potential alternative to antibiotic growth promoters in livestock feed. The beneficial effects of dietary inclusion of turmeric on growth performance and digestibility have been reported [32,33]. However, limited studies are available on the influence of turmeric on the gut microbiota of pigs. Furthermore, to the best of our knowledge, there is no metagenomic study on the effects of turmeric supplemented diets on gut microbiota in pigs. Therefore, the objective of this study was to investigate the effect of dietary turmeric supplementation on growth performance, blood parameters, fecal score, fecal SCFAs, branchedchain fatty acids (BCFAs), gut microbiota, and histomorphology of the ileum in weaned piglets. 


\section{MATERIALS AND METHODS}

\section{Experimental design, animal, diet, and housing}

A total of 48 newly weaned piglets (Duroc $\times$ [Landrace $\times$ Yorkshire]) with an initial average bodyweight (BW) of $7.35 \pm 0.3 \mathrm{~kg}$ were used in 6-week feeding trail. All piglets were obtained from one farm and weaned at 28 days of age. Animal experiments were performed at the Animal Research Center at Chungnam National University, Daejeon, Korea. Animal care procedures and experimental protocols were approved by the Animal Care and Use Committee of Chungnam National University (Approval\# CNU-00611). All piglets were randomly assigned to two dietary treatments: the group fed with basal diet only (control) and the basal diet supplemented with $1 \%$ (w/w) turmeric powder (turmeric). Turmeric powder was purchased from a local supermarket in Seoul, Korea. Each dietary treatment had four replicates per treatment, with six piglets per pen. In total, 48 male piglets, 24 piglets in the control group, and 24 piglets in the turmeric group were allotted. Diets in mash form were formulated to meet the requirements suggested by the NRC 2012 [34]. Nutrient composition of the diet and chemical composition of turmeric are shown in Tables 1 and 2, respectively. A general maintenance program was used for sows and piglets during lactation. The diets did not include any antibiotics to avoid antibacterial activity during the lactation

Table 1. Nutrient composition of basal diet fed to experimental piglets

\begin{tabular}{|c|c|c|}
\hline Ingredient (\%) & Phase $1^{1)}$ & Phase $2^{2)}$ \\
\hline Corn & 31.57 & 51.56 \\
\hline Soybean meal $(44 \% \mathrm{CP})$ & 18.00 & 26.56 \\
\hline Soy protein concentrate & 16.96 & 8.00 \\
\hline Dried whey & 24.00 & 10.00 \\
\hline Lactose & 4.00 & - \\
\hline Soybean oil & 3.00 & 1.35 \\
\hline Limestone & 1.00 & 1.00 \\
\hline Monocalcium phosphate & 0.90 & 0.90 \\
\hline Vitamin pre-mix ${ }^{3)}$ & 0.20 & 0.20 \\
\hline Mineral pre-mix ${ }^{4}$ & 0.20 & 0.20 \\
\hline L-Lysine-HCl & 0.08 & 0.17 \\
\hline DL-Methionine & 0.09 & 0.07 \\
\hline Total & 100 & 100 \\
\hline \multicolumn{3}{|c|}{ Calculated energy and nutrient content } \\
\hline ME (Mcal/kg) & 3.53 & 3.42 \\
\hline $\mathrm{CP}(\%)$ & 24.49 & 22.51 \\
\hline Calcium (\%) & 0.81 & 0.73 \\
\hline Phosphorus (\%) & 0.69 & 0.63 \\
\hline Lysine (\%) & 1.54 & 1.41 \\
\hline
\end{tabular}

Week 1 to 3 (21 days).

${ }^{2}$ Week 4 to 6 (21 days).

${ }^{3)}$ Provided per kilogram of diet: vitamin $\mathrm{A}, 12,000 \mathrm{IU}$; vitamin $\mathrm{D}_{3}, 2,500 \mathrm{IU}$; vitamin $\mathrm{E}, 30 \mathrm{IU}$; vitamin $\mathrm{K}_{3}, 3 \mathrm{mg}$; D-pantothenic acid, $15 \mathrm{mg}$; nicotinic acid, $40 \mathrm{mg}$; choline, $400 \mathrm{mg}$; and vitamin $\mathrm{B}_{12}, 12 \mu \mathrm{g}$.

${ }^{4)} \mathrm{Fe}, 90 \mathrm{mg}$ from iron sulfate; $\mathrm{Cu}, 8.8 \mathrm{mg}$ from copper sulfate; $\mathrm{Zn}, 100 \mathrm{mg}$ from zinc oxide; $\mathrm{Mn}, 54 \mathrm{mg}$ from manganese oxide; I, $0.35 \mathrm{mg}$ from potassium iodide; $\mathrm{Se}, 0.30 \mathrm{mg}$ from sodium selenite.

The calculation for the energy and nutrient contents was performed using the below formula:

Calculated enery or each nutrient content $=$ sum of (energy or each nutrient value of each ingredient used in a diet $\times \%$ concentration of each ingredient used in a diet / 100).

$\mathrm{ME}$, metabolizable energy; $\mathrm{CP}$, crude protein. 
Table 2. Chemical composition of turmeric powder

\begin{tabular}{lc}
\hline \multicolumn{1}{c}{ Constituents } & Quantity (\%) \\
\hline Moisture & 10.86 \\
Crude protein & 37.39 \\
Crude fat & 2.78 \\
Crude fiber & 3.11 \\
Crude ash & 6.26 \\
Carbohydrates & 42.71 \\
Starch & 35.91 \\
Neutral detergent fiber (NDF) & 12.11 \\
Acid detergent fiber (ADF) & 9.68 \\
Soluble dietary fiber (SDF) & 2.24 \\
Insoluble dietary fiber (ISDF) & 17.37 \\
Cellulose & 8.77 \\
$\beta$-Glucans & 13.04 \\
Lignin & 0.91 \\
Hemicellulose & 2.43 \\
\hline
\end{tabular}

and experimental periods. All experimental piglets were housed in an environmentally controlled, slatted-floor facility with a mechanical ventilation system. Each pen was equipped with a self-feeder and nipple water to allow ad libitum access to feed and water throughout the experimental period. The piglets were individually weighed at the start and at weeks 3 and 6 of the experimental period, and feed intake was recorded throughout the experiment to calculate average daily gain (ADG), average daily feed intake (ADFI), and the gain-to-feed ratio (G:F) was calculated using ADG and ADFI.

\section{Sample collection}

On the final day of the experiment, freshly voided fecal samples from one randomly selected piglet in each pen were collected by rectal stimulation for $16 \mathrm{~S}$ sequencing and SCFA analysis. The number of samples was determined based on our previous pilot study (data not shown). All samples were stored at $-80^{\circ} \mathrm{C}$ until analysis. Blood samples were collected aseptically through an external jugular vein puncture.

\section{Serum hematological and biochemical indices}

All blood samples were centrifuged at 1,200xg for $10 \mathrm{~min}$ at $4^{\circ} \mathrm{C}$, and plasma and serum samples were sent to Neodin Vet Lab (Seoul, Korea) on the same day of sample collection for analysis of concentrations of total proteins, creatine, urea, glucose, total cholesterol, triglycerides, and gammaglutamyl transferase. Plasma samples were analyzed for red blood cell (RBS), white blood cell (WBC), and platelet counts.

\section{Volatile fatty acids analysis}

Volatile fatty acids (VFA) analysis was performed according to Cho et al. [35] with modifications using gas chromatography (GC) (6890 N, Agilent, Santa Clara, CA, USA), equipped with an HP-INNOWAX column and a flame ionization detector. Fresh fecal samples $(1 \mathrm{~g})$ were acidified with $1 \mathrm{~mL}$ of $25 \%$ phosphoric acid solution, $3 \mathrm{~mL}$ of distilled water, and $50 \mu \mathrm{L}$ saturated mercury solution (Sigma-Aldrich, St. Louis, MO, USA). After $30 \mathrm{~min}$, the samples were centrifuged at $3,000 \times \mathrm{g}$ for $20 \mathrm{~min}$, and $3 \mathrm{~mL}$ of the supernatant was collected. Then, the $3 \mathrm{~mL}$ of the supernatant 
was centrifuged at $13,800 \times \mathrm{xg}$ for $10 \mathrm{~min}$ and filtered through a $0.2 \mu \mathrm{m}$ filter (Whatman, Uppsala, Sweden). The filtrates were mixed with an equal amount of methanol and then placed in $2.0 \mathrm{~mL}$ $\mathrm{GC}$ vials (Agilent) to measure the concentration of volatile fatty acids. The sample injection volume was $2 \mu \mathrm{L}$, with a split ratio of 10:1.

\section{Histometric analysis of piglet ileum}

On the final day of the experimental period, a total of 8 piglets (4 piglets per group) were slaughtered, and the intestinal tract was removed. The distal ileum segments were collected and fixed with $4 \%$ paraformaldehyde in $0.01 \mathrm{M}$ phosphate buffered saline. The ileum sections were stained with hematoxylin and eosin for microscopic examination to determine villus height $(\mathrm{VH})$, crypt depth (CD), VH:CD, villus width, villus area, and number of goblet cells.

\section{Occurrence of diarrhea}

The diarrhea score of each piglet was recorded at weeks 1 to 6 of the trial. Diarrhea was assessed visually based on consistency of the feces, and fecal scores were determined using the following fecal scoring system: 1 hard, dry pellet; 2 firm, formed stool; 3 soft, moist stool that retains shape; 4 soft, unformed stool that assumes shape of container; 5 watery liquid that can be poured. The fecal score was assessed in a treatment-blinded manner by two trained individuals. Scores were recorded on a pen-basis observation of individual piglets and signs of stool consistency in the pen [36].

\section{DNA extraction and sequencing}

Total DNA was extracted from fecal samples using the PowerSoil ${ }^{\circledR}$ DNA Isolation Kit according to the manufacturer's protocol. The quantification of DNA and DNA quality was measured using PicoGreen and Nanodrop (Thermo Scientific, Waltham, MA, USA). The primers used for 16S V3V4 rRNA gene amplification are listed in Table 3.

Input gDNA (12.5 ng) was amplified with 16S V3-V4 primers, and a subsequent limitedcycle amplification step was performed to add multiplexing indices and Illumina sequencing adapters. Amplicons from PCR were pooled using PicoGreen and used as input for Illumina library preparation. The size of the libraries was verified using the LabChip GX HT DNA High Sensitivity Kit (PerkinElmer, Waltham, MA, USA). Samples were sequenced using an Illumina MiSeq (Macrogen, Seoul, Korea).

\section{Sequence read processing and data analysis}

Sequencing reads obtained from Illumina MiSeq were filtered and trimmed using CD-HITOUT software and rDNA Tools [37]. To perform taxonomic assignment, operative taxonomic units (OTUs) were selected based on a 97\% threshold of sequence similarity using the QIIMEUCLUST program. The filtered reads were clustered and OTUs were generated using CD-HITDUP. The sequences that passed from the quality filters were analyzed using the QIIME pipeline, which included features to calculate diversity indices and phylogenetic diversity (PD) rarefaction curves. Alpha-diversity indices including OTUs, Shannon, Chao1, and Simpson index were measured for each sample, and beta-diversity of the two groups were illustrated using principal

Table 3. Primers used for 16S V3-V4 rRNA gene amplification

\begin{tabular}{ll}
\hline \multicolumn{1}{c}{ Direction } & \multicolumn{1}{c}{ Primer } \\
\hline Forward & 5'TCGTCGGCAGCGTCAGATGTGTATAAGAGACAGCCTACGGGNGGCWGCAG \\
Reverse & 5'GTCTCGTGGGCTCGGAGATGTGTATAAGAGACAGGACTACHVGGGTATCTAATCC \\
\hline
\end{tabular}


component analysis (PCA) and principal coordinate analysis (PCoA) based on weighted UniFrac distances. The Ribosomal Database Project (RDP) classifier was used for taxonomic assignment of the fecal microbiome of the two groups.

\section{Statistical analysis}

Data of hematological and biochemical analyses, VFA concentrations, histometric analysis, and microbial diversity indices were analyzed by Student's $t$-test using the SPSS Statistics Version 23 software package (IBM, Armonk, NY, USA). Growth performance data were statistically analyzed using the GLM procedure of the Statistical Analysis System (SAS Institute, Cary, NC, USA). The Mann-Whitney $U$ test was used to determine the statistical significance of the relative abundance of microbial communities in two groups at the phylum, class, genus, and species levels (SPSS version 23, IBM). Statistical significance was reported at $p<0.05$, and trends were noted when 0.05 $<p<0.10$.

\section{RESULTS}

\section{Effects of turmeric on growth performance and fecal score analysis}

In the current study, piglets supplemented with turmeric diet had increased final BW compared to piglets fed the control diet (Table 4). Furthermore, dietary supplementation with turmeric had significant effects on ADFI and tendency effects on G:F at week 3 without affecting ADG. At week 6, increased ADG $(p=0.026)$ and tendency effects on G:F $(p=0.09)$ did not affect ADFI. Overall, turmeric supplementation had increased $(p<0.04)$ ADG and tendency toward $\mathrm{G}: \mathrm{F}(p=0.078)$ without affecting total ADFI $(p=0.349)$. The diarrhea incidence scores are presented in Table 5. During the experimental period, none of the piglets suffered from diarrhea. Supplementation of turmeric with the basal diet improved the fecal score during week 6 ( $p=$ 0.009).

Table 4. Growth performance of weaned piglets fed control and turmeric supplemented diets

\begin{tabular}{lrrrr}
\hline \multicolumn{1}{c}{ Items } & Control & Turmeric & SEM & $p$-value \\
\hline BW (kg) & & & & \\
Initial & 7.33 & 7.36 & 0.038 & 0.392 \\
Wk 3 & 16.64 & 17.40 & 0.275 & 0.115 \\
Wk 6 & 24.96 & 26.08 & 0.29 & 0.029 \\
Phase 1 (wk 1-3) & & & & \\
ADG (g) & 443 & 478 & 12.87 & 0.14 \\
ADFI (g) & 357 & 336 & 5.247 & 0.042 \\
G:F & 1.241 & 1.424 & 0.046 & 0.06 \\
Phase 2 (wk 4-6) & & & & 0.026 \\
ADG (g) & 394 & 414 & 6.945 & 0.158 \\
ADFI (g) & 822 & 780 & 17.087 & 0.09 \\
G:F & 0.480 & 0.532 & 0.071 & \\
Total & & & & 0.042 \\
ADG (g) & 419 & 446 & 6.943 & 0.349 \\
ADFI (g) & 693 & 674 & 15.827 & 0.078 \\
G:F & 0.605 & 0.664 & 0.016 & \\
\hline
\end{tabular}

Control, basal diet; Turmeric, basal diet with $1 \%$ (w/w\%) of turmeric powder; BW, body weight; ADG, average daily gain; ADFI, average daily feed intake; G:F, gain-to-feed ratio. 
Table 5. Effects of turmeric supplementation on fecal score of piglets

\begin{tabular}{rcccc}
\hline Items & Control & Turmeric & SEM & $p$-value \\
\hline Fecal score ${ }^{1)}$ & & & & \\
Week 1 & 3.29 & 3.50 & 0.10 & 0.22 \\
Week 2 & 3.43 & 3.24 & 0.06 & 0.08 \\
Week 3 & 3.29 & 3.21 & 0.06 & 0.22 \\
Week 4 & 3.40 & 3.50 & 0.05 & 0.03 \\
Week 5 & 3.41 & 3.24 & 0.04 & 0.08 \\
Week 6 & 3.51 & 3.36 & 0.05 & 0.009 \\
\hline
\end{tabular}

${ }^{1)}$ Fecal scores were determined using the following fecal scoring system: 1 hard, dry pellet; 2 firm, formed stool; 3 soft, moist stool that retains shape; 4 soft, unformed stool that assumes shape of container; 5 watery liquid that can be poured.

\section{Effect of dietary turmeric on hematological and biochemical indices}

The hematological parameters for the turmeric and control groups are shown in Table 6. Dietary turmeric supplementation did not influence leukocyte, erythrocyte, and thrombocyte counts, mean corpuscular volume $(\mathrm{MCV})$, mean corpuscular hemoglobin $(\mathrm{MCH})$, and mean corpuscular hemoglobin concentration (MCHC). There were no significant differences between the two groups $(p>0.05)$. The effects of dietary turmeric on the biochemical variables are summarized in Table 7. Turmeric supplementation did not significantly affect the levels of total, LDL, and HDL cholesterol. Numerically, HDL-cholesterol was higher in the turmeric group, but not significantly $(p=0.776)$.

\section{VFA analysis}

As shown in Table 8, dietary turmeric supplementation increased SCFA production $(p<0.05)$. Acetic, propionic, and butyric acids were predominant. The highest acetic acid levels were observed in the turmeric group, followed by propionic and butyric acids. The levels of isobutyric and isovaleric acids were not significantly different between the two groups; however, the concentrations showed

Table 6. Effect of turmeric supplementation on hematological parameters in piglets

\begin{tabular}{|c|c|c|c|c|}
\hline Items & Control & Turmeric & SEM & $p$-value \\
\hline \multicolumn{5}{|l|}{ Leukocytes } \\
\hline White blood cell $(\mathrm{K} / \mu \mathrm{L})$ & 19.27 & 14.69 & 1.62 & 0.18 \\
\hline Neutrophil (\%) & 43.8 & 31.03 & 4.51 & 0.23 \\
\hline Lymphocyte (\%) & 49.03 & 61.27 & 3.98 & 0.2 \\
\hline Monocyte (\%) & 4.7 & 4.77 & 0.77 & 0.97 \\
\hline Eosinophil (\%) & 4.1 & 2.97 & 0.66 & 0.45 \\
\hline \multicolumn{5}{|l|}{ Erythrocytes } \\
\hline Red blood cell $\left(\mathrm{M} / \mathrm{mm}^{3}\right)$ & 6 & 5.8 & 0.14 & 0.54 \\
\hline Hemoglobin (g/dL) & 10.8 & 10.33 & 0.29 & 0.48 \\
\hline \multicolumn{5}{|l|}{ Thrombocytes } \\
\hline Platelet $(\mathrm{K} / \mu \mathrm{L})$ & 392.67 & 450.67 & 101.92 & 0.81 \\
\hline MCV (fl) & 63.6 & 62.17 & 0.77 & 0.41 \\
\hline $\mathrm{MCH}(\mathrm{pg})$ & 18.03 & 17.83 & 0.41 & 0.84 \\
\hline $\mathrm{MCHC}(\%)$ & 28.43 & 28.63 & 0.82 & 0.92 \\
\hline
\end{tabular}

$\mathrm{MCV}$, mean corpuscular volume; $\mathrm{MCH}$, mean corpuscular heamoglobin concentration; $\mathrm{MCHC}$, mean corpuscular heamoglobin concentration. 
Table 7. Effect of turmeric supplementation on biochemical parameters in piglets

\begin{tabular}{lrrrc}
\hline \multicolumn{1}{c}{ Items } & Control & Turmeric & SEM & p-value \\
\hline TP (g/dL) & 7.15 & 5.875 & 0.67 & 0.38 \\
ALB (g/dL) & 3.7 & 3.575 & 0.07 & 0.418 \\
T.Bil (mg/dL) & 0.3 & 0.2 & 0.04 & 0.272 \\
Glucose (mg/dL) & 100.25 & 81 & 9.0 & 0.321 \\
BUN (mg/dL) & 5.475 & 4.85 & 0.39 & 0.463 \\
Creatinine (mg/dL) & 0.735 & 0.8925 & 0.05 & 0.164 \\
Y-GTP (U/L) & 45.75 & 39.5 & 4.82 & 0.554 \\
LDH (U/L) & 1033.75 & 793.75 & 203.81 & 0.596 \\
Chol (mg/dL) & 93.75 & 97.75 & 4.03 & 0.668 \\
TG (mg/dL) & 52.25 & 53.5 & 2.06 & 0.787 \\
HDL (mg/dL) & 29.675 & 31.525 & 2.9 & 0.776 \\
LDL (mg/dL) & 41.9 & 52.7 & 4.37 & 0.244 \\
AST (U/L) & 113 & 108 & 24.73 & 0.928 \\
ALT (U/L) & 79.75 & 76.75 & 3.19 & 0.678 \\
\hline
\end{tabular}

TP, total protein; ALB, albumin; T.Bil, total bilirubin; BUN, blood urea nitrogen; GTP, glutamyl transpeptidase; LDH, lactic acid dehydrogenase; Chol, cholesterol; TG, triglyceride; HDL, high density lipoprotein; LDL, low density lipoprotein; AST, aspartate amino transferase; ALT, alanine aminotransferase.

Table 8. Effects of turmeric diet on fecal volatile fatty acids (SCFAs and BCFAs) concentration.

\begin{tabular}{lcccc}
\hline \multicolumn{1}{c}{ Concentration $(\boldsymbol{\mu g} / \mathbf{g})$} & Control & Turmeric & SEM & $\boldsymbol{p}$-value \\
\hline Acetic acid & 5.56 & 9.8 & 0.97 & 0.011 \\
Propionic acid & 2.5 & 4.51 & 0.4 & 0.001 \\
Butyric acid & 1.88 & 3.87 & 0.41 & 0.001 \\
Valeric acid & 0.61 & 0.96 & 0.08 & 0.022 \\
Iso butyric acid & 0.55 & 0.74 & 0.05 & 0.057 \\
Iso valeric acid & 0.83 & 1.22 & 0.11 & 0.057 \\
Total SCFA & 10.57 & 19.15 & 1.78 & 0.002 \\
Total BCFA & 1.38 & 1.96 & 0.16 & 0.056 \\
\hline
\end{tabular}

SCFA, short-chain fatty acids; BCFA, branched-chain fatty acids.

an increasing tendency in tumeric group $(p=0.057)$.

\section{Effects of dietary treatments on ileum morphology of weaned piglets}

Turmeric supplementation had no effect $(p>0.05)$ on VH and CD (Table 9). However, the $\mathrm{VH}: \mathrm{CD}$ ratios were higher in the turmeric group than in the control group $(p=0.04)$. There was no difference in the surface area and width of villi in the turmeric group compared to the control group.

\section{DNA sequence data and bacterial diversity}

A total of 437,000 read bases were obtained from the sequencing of fecal samples from the control and turmeric groups. After filtering and removing low-quality sequences, an average of 39,289 and 41,318 reads were obtained for control and turmeric group samples, respectively. A total of 870 OTUs belonging to bacteria and archaea were identified at the 97\% threshold level.

Alpha-diversity analyses, including Shannon, Simpson, and Chao1 indices were analyzed to explore the effect of dietary turmeric on the richness and evenness of gut microbiota. The 
Table 9. Effects of turmeric supplementation on ileum morphology

\begin{tabular}{lrrrc}
\hline \multicolumn{1}{c}{ Item } & Control & Turmeric & SEM & $p$-value \\
\hline Villus height $(\mu \mathrm{m})$ & 396.77 & 409.70 & 16.65 & 0.596 \\
Crypt depth $(\mu \mathrm{m})$ & 281.47 & 245.33 & 13.74 & 0.096 \\
VH:CD & 1.42 & 1.68 & 0.08 & 0.040 \\
Villus width $(\mu \mathrm{m})$ & 143.62 & 169.88 & 11.65 & 0.145 \\
Villus area $\left(\mu \mathrm{m}^{2}\right)$ & 30,919 & 36,862 & 3,571 & 0.270 \\
Number of goblet cells & 15.67 & 13.83 & 1.17 & 0.246 \\
\hline
\end{tabular}

$\mathrm{VH}: \mathrm{CD}$, villus height-to-crypt depth ratio.

$\alpha$-diversity metrics are shown in Figs. 1A, B, and C. According to the data, all diversity indices were not significantly different $(p>0.05)$ between groups, while turmeric administration showed a decreasing tendency in the Shannon index $(p=0.055)$.

$\alpha$-Diversity rarefaction curves based on observed OTUs, PD whole tree, and sequence for samples between the two groups indicated sufficient sequencing depth (Figs. 2A and B). PCoA based on weighted Unifrac distance showed two clusters containing each sample of both control

A

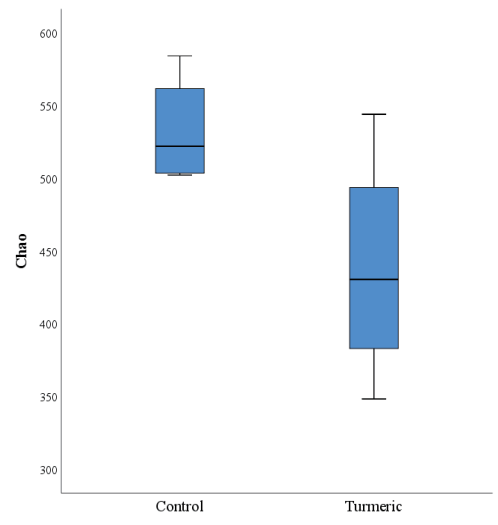

B

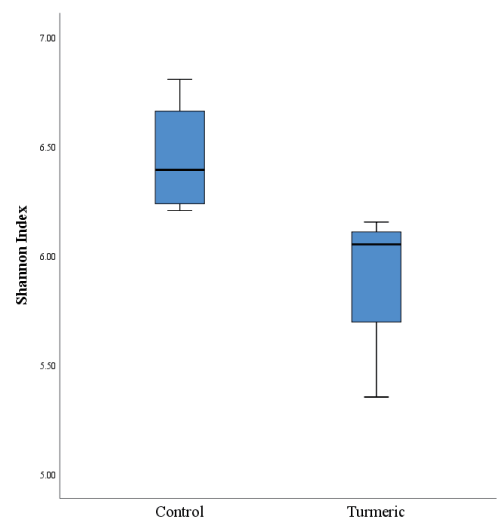

C

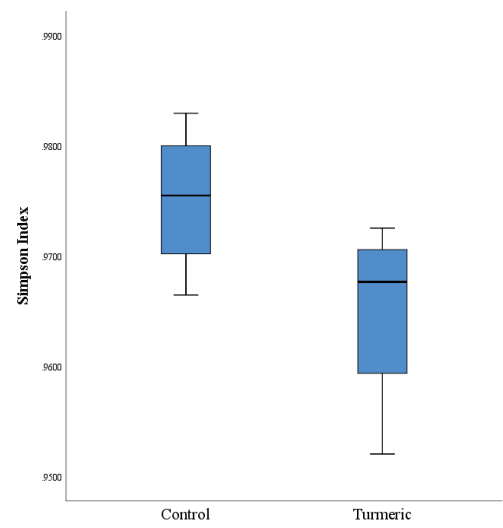

Fig. 1. Microbial diversity indices for control and turmeric fed piglets. (A) The Chao value of control and turmeric groups. (B) The Shannon index of control and turmeric groups. (C) The Simpson index of control and turmeric groups.

A

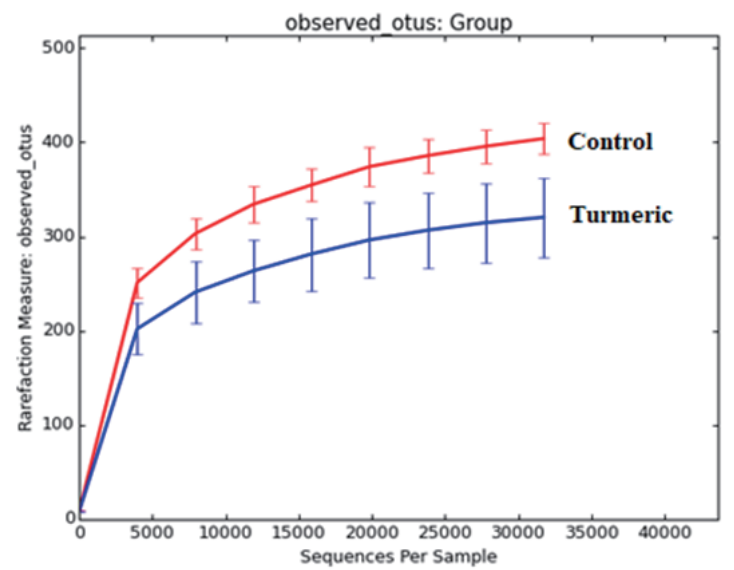

B

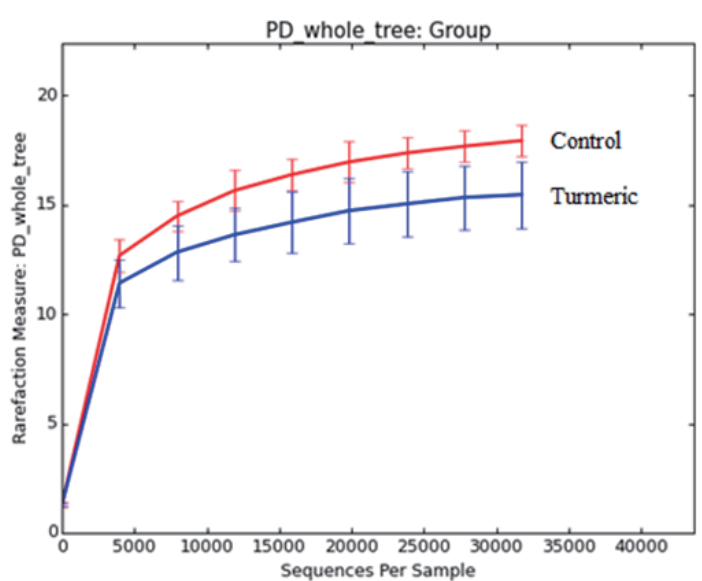

Fig. 2. $\alpha$-Diversity rarefaction curves. (A) Rarefaction curves represent the number of sequences per sample against the number of observed OTUs in control and turmeric groups. (B) Rarefaction curves of PD whole tree in control and turmeric groups. OTUs, operative taxonomic units; PD, phylogenetic diversity. 
and turmeric groups, except for one sample in each group (Fig. 3).

\section{Taxonomic analysis}

The effects of turmeric supplementation on fecal microbial composition were observed at different taxonomic levels. At the phylum level, 10 phyla were observed in each sample. Bacteroidetes and Firmicutes were the predominant phyla, accounting for $90 \%$ of the total relative abundance. The Firmicutes abundance ratio was similar in the control (51.8\%) and turmeric (52.46\%) groups (Fig. 4). Bacteroides abundance decreased in turmeric (27.62\%) fed piglets than in control (39.61\%) fed piglets $(p=0.021)$.

As shown in Fig. 5, Bacteroidia and Clostridia were the predominant classes in both the control and treatment groups. Twelve classes were identified. The relative abundance of Bacteroidia was

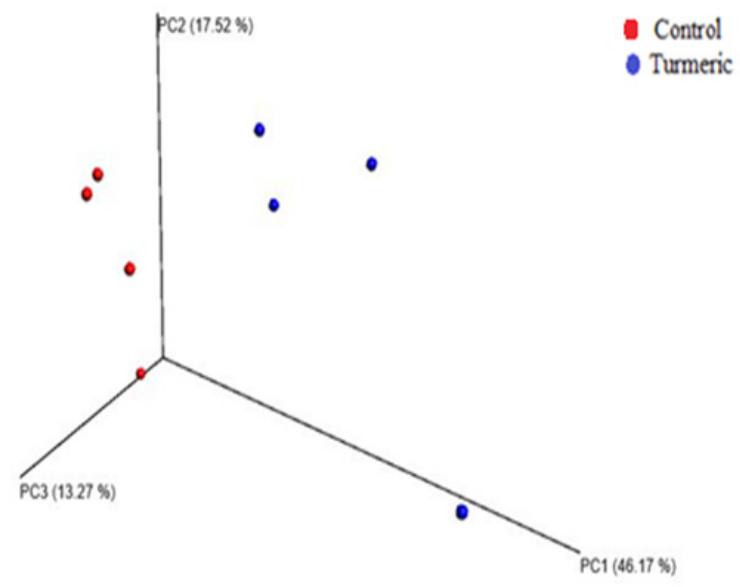

Fig. 3. PcoA analysis of control and turmeric groups. Three dimentional plot based on weighted UniFrac distances. PCoA, principal coordinate analysis.

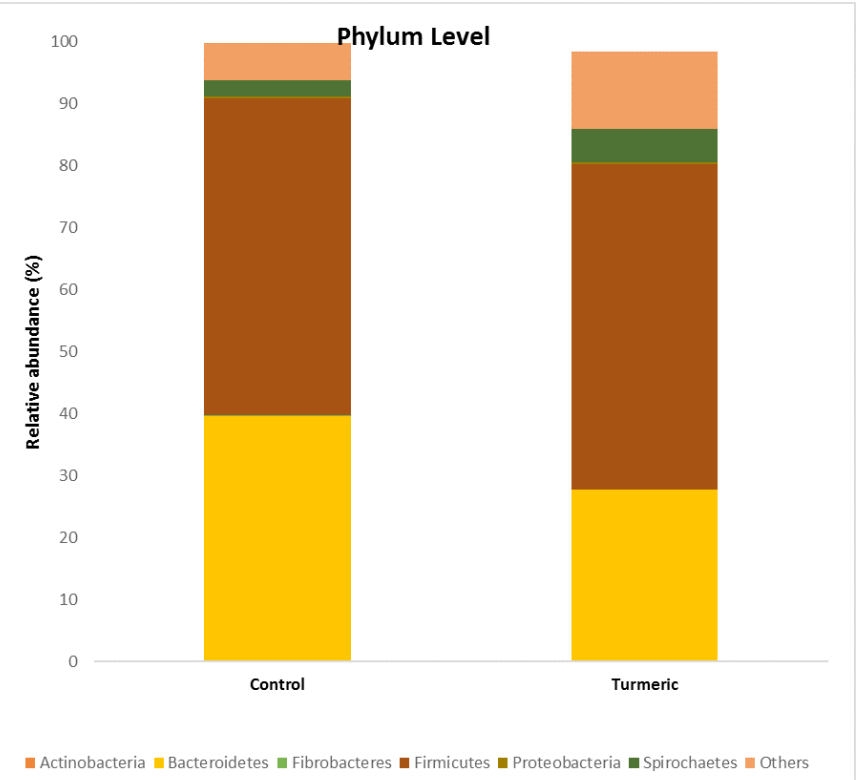

Fig. 4. Bacterial composition and abundance ratio of the fecal microbiota of piglets at phylum level. 


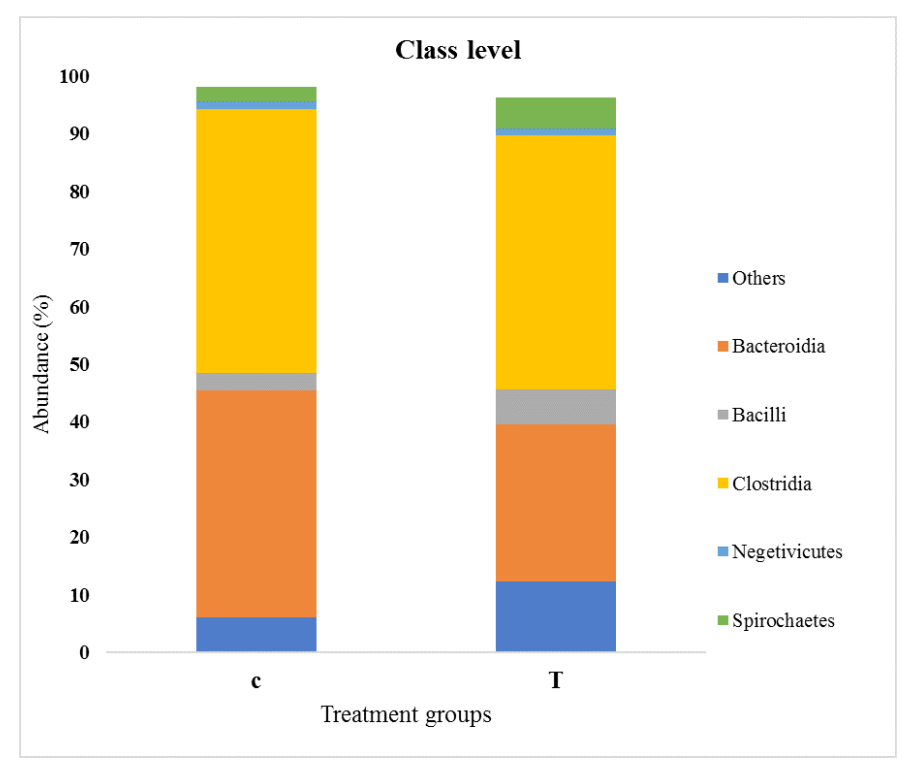

Fig. 5. Bacterial composition and abundance ratio of the fecal microbiota of piglets at class level. C, control group; T, turmeric group.

significantly decreased in turmeric-fed piglets $(p=0.021)$. However, the remaining bacterial abundance was not affected by the turmeric diet.

At the genus level, approximately 105 genera were found in fecal samples from both dietary groups. The 15 most abundant genera in the two groups are shown in Fig. 6. Among the abundant genera, eight belonged to Firmicutes, 6 from Bacteroidetes, and 1 from Spirochaetes. The unclassified bacteria at the genus level were higher in the turmeric group than in the control group $(p=0.043)$. The relative abundance of Prevotella genera was significantly lower in the turmeric group than in the control group $(p=0.021)$. The Lactobacillus genus showed an increasing trend in the turmeric group $(p=0.083)$. The remaining genera did not differ significantly between the two groups. At the

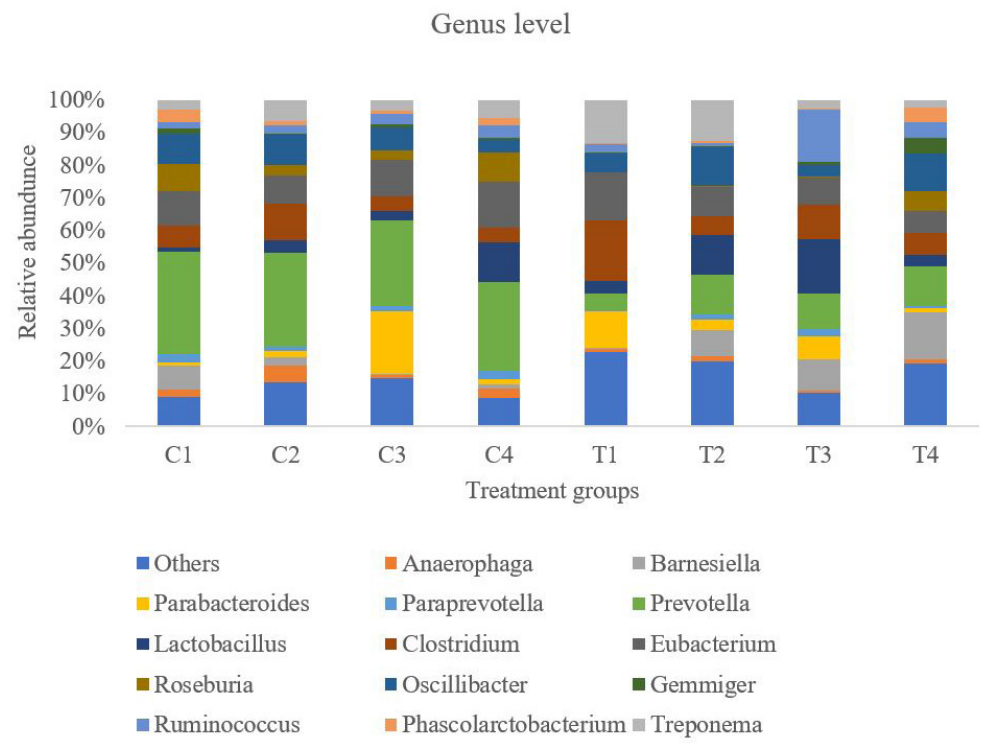

Fig. 6. Bacterial composition and abundance ratio of the fecal microbiota of piglets at genus level. C, control group; T, turmeric group. 
Table 10. Effects of turmeric supplementation on fecal microbiota at species level

\begin{tabular}{|c|c|c|c|c|c|}
\hline \multirow{2}{*}{ Phylum } & \multirow{2}{*}{ Genus } & \multirow{2}{*}{ Species } & \multicolumn{2}{|c|}{ Abundance ratio (\%) } & \multirow{2}{*}{$p$-value } \\
\hline & & & Control & Turmeric & \\
\hline Bacteroidetes & Bacteroides & Bacteroides stercoris & 1.28 & 0.32 & 0.021 \\
\hline Bacteroidetes & Prevotella & Prevotella shahii & 4.33 & 0.51 & 0.043 \\
\hline Bacteroidetes & Prevotella & Prevotella timonensis & 1.89 & 0.1 & 0.021 \\
\hline Bacteroidetes & Prevotella & Prevotella oris & 2.01 & 1.21 & 0.021 \\
\hline Bacteroidetes & Muribaculum & Muribaculum intestinale & 13 & 6.71 & 0.083 \\
\hline Firmicutes & Lactobacillus & Lactobacillus reuteri & 0.43 & 3.38 & 0.021 \\
\hline Firmicutes & Falcatimonas & Falcatimonas natans & 0.24 & 0.59 & 0.021 \\
\hline Firmicutes & Clostridium & Clostridium bornimense & 0.06 & 0.26 & 0.021 \\
\hline Firmicutes & Peptococcus & Peptococcus simiae & 0 & 0.2 & 0.018 \\
\hline Firmicutes & Negativebacillus & Negativibacillus massiliensis & 0.05 & 0.15 & 0.021 \\
\hline Spirochaetes & Treponema & Treponema berlinense & 0.65 & 2.38 & 0.059 \\
\hline
\end{tabular}

species level, a total of nine species abundance ratios were statistically significant between the two groups, including Lactobacillus spp. (Table 10).

\section{DISCUSSION}

Turmeric root powder is commonly used for medicinal purposes and as a spice in traditional cooking. A couple of studies have investigated the effects of turmeric supplementation on the growth performance of piglets [32,33]. However, more studies are needed to explore the effects of turmeric on intestinal morphology and gut microbial communities. Gut microbiota provides not only fuel to colonocytes, but also helps in immune system development and maintenance of intestinal homeostasis [10]. In this study, we describe the impact of dietary turmeric on growth performance, gut morphology, and microbiota in a porcine model. Turmeric inclusion in piglet diets improved ADG and showed higher average final BW and lower feed intake than the control group. Similarly, a previous study indicated that diets containing turmeric powder at $2 \%, 4 \%$, and 6\% improved final live weight and feed conversion ratio compared with basal diet fed pigs [38]. Maneewan et al. [32] reported that the effects of low levels of dietary turmeric supplementation on nursery pigs at doses of $0.05 \%, 0.10 \%$, and $0.20 \%$ did not influence ADFI, ADG, and feed efficiency. The beneficial effects of turmeric on growth performance might be due to the enhanced secretion of amylase, trypsin, chymotrypsin, and lipase enzymes [38]. Similarly, Singh et al. [39] reported that $1 \%$ dietary turmeric resulted in increased BW gain in broiler chickens, however, turmeric did not influence the feed efficiency in this study. Tubcharoen et al. [40] reported similar results in growing-finishing pigs. Furthermore, in this study, fecal scores were much higher in the control group. The incidence of diarrhea among piglets was reduced when the diet was supplemented with turmeric.

Hematological and biochemical variables were determined to determine the health and stress status of the animals. There were no significant differences in the values of RBC, WBC, MCV, $\mathrm{MCHC}, \mathrm{MCH}$, and platelets. However, all hematological parameters were within the normal range for swine [41]. This indicates that turmeric causes no variations in the hematology of piglets and that none of the animals experienced stress during the experimental period. The serum biochemical variables were not affected by turmeric. The main active compound, curcumin, in turmeric exhibits hypocholesterolemic activity. Curcumin showed beneficial effects in high-fat fed animals [42]. 
In the present study, turmeric did not influence cholesterol levels in piglets. These results contrast with those of a previous study that showed that curcumin supplementation decreased serum LDL cholesterol levels in weaned piglets [2]. The lipid-lowering effects of turmeric and curcuminoids are associated with the dose and solubility of curcumin. Considering the poor solubility and bioavailability of curcumin, Porn-anek et al. [43] developed a carrier-based turmeric oleoresin using a solid dispersion technique to enhance curcumin solubility. Pigs fed with the newly developed turmeric oleoresin had increased HDL cholesterol and lowered LDL cholesterol, total cholesterol, and triglycerides.

Intestinal metabolites such as SCFAs play a major role in the regulation of gut homeostasis [42]. SCFAs are the end products produced by bacterial fermentation of non-digestible carbohydrates in the colon [13,44-46]. Primary SCFAs are acetic, propionic, and butyric acids, with butyric acid being the main energy substrate for colonic epithelial cells $[44,47]$. In this study, turmeric dietfed piglets produced higher levels of SCFAs. Acetic, propionic, and butyric acid concentrations were higher among all the SCFAs. Catabolism of turmeric polysaccharides provides energy for fermentative bacteria such as Lactobacillus spp. and Clostridium spp., which results in an increase in SCFA production [28]. Similarly, Han et al. [48] reported that in vitro fermentation of spent turmeric powder with pig fecal bacteria resulted in higher concentrations of acetate and propionate. Microbial abundance, particularly Lactobacillus abundance in the gut, is associated with gut SCFA production. The status of gut health can be determined by intestinal morphology, such as $\mathrm{VH}$, $\mathrm{CD}$, and the VH:CD ratio. Villi plays a major role in increasing nutrient absorption, especially in the small intestine $[12,49]$. In this study, turmeric-fed piglets showed a higher VH:CD ratio than the control group. Increased VH:CD ratios indicate improved nutrient absorption function [50]. This result is consistent with a previous study that piglets consuming dietary curcumin showed an improved VH:CD ratio [49].

Turmeric has been reported to be an antimicrobial and anti-inflammatory agent $[21,22]$. Avanço et al. [51] reported that $\alpha$-turmerone, $\beta$-turmerone, and ar-turmerone components of turmeric showed antifungal, antimycotoxigenic, and antioxidant activities. Thus, turmeric could alter microbial communities in the intestine by inhibiting pathogenic bacteria. The taxonomic analysis in this study showed that Firmicutes and Bacteroidetes were the most abundant phyla in both groups of piglet gut microbiota, as reported in previous studies [52,53]. The abundance ratio of Bacteroidetes decreased in turmeric-fed piglets. However, Firmicutes phyla were not altered by turmeric. Bacteroidetes are gram-negative anaerobic bacteria normally present in the intestinal flora. Bacteroides are generally beneficial to the host through their metabolism of dietary polysaccharides; however, Bacteroidetes are involved in inflammatory pathology when the gut microbiota is in an imbalanced state [54,55]. Moreover, Zhao et al. [56] reported that fecal Bacteroides were negatively correlated with SCFAs and amino acids in mice. We also observed a decreased abundance of Bacteroidetes and increased concentrations of SCFAs in turmeric-fed piglets. Hence, low Bacteroides and higher SCFAs are predicted to promote the gut health of piglets in the post-weaning period. Turmeric had no impact on the overall microbial diversity and richness, except for the lower trend of the Shannon index. Similarly, Shen et al. [57] reported that oral administration of curcumin tended to decrease microbial diversity and richness with no significant differences. Despite no significant differences in microbial diversity, the abundance of specific bacteria, including Lactobacillus and Prevotella, were altered in turmeric fed piglets. At the genus level, Prevotella genera decreased in the turmeric group, similar to previous studies [57]. Little is known about the role of Prevotella in health promotion. Like other bacteria in normal microflora, Prevotella spp. act as opportunistic pathogens and have been associated with infections [58,59]. Turmeric increased the abundance of Lactobacillus spp. and Clostridium spp. and decreased the abundance of Prevotella spp. Similarly, Han et al. [48] reported 
that spent turmeric fermented with swine microbiota showed increased Lactobacillus populations compared to other groups. Kosti et al. [60] also observed higher Lactobacillus counts and lower $E$. coli counts in turmeric-fed hens than in the basal diet group. Some studies have suggested that the phenolic compound curcumin in turmeric root powder possesses alterations in gut microbial composition [28]. Moreover, curcumin has been found to improve the barrier function of the intestine by modulating intracellular signaling pathways [61]. The results obtained from this study revealed that dietary turmeric influences gut microbial fermentation and improves gut health by enhancing beneficial bacteria, SCFAs, and gut morphology. However, in the current study, we used whole turmeric root powder as a dietary supplement. Further studies that use turmeric extract or curcumin alone are needed to clarify the microbial alteration effects in the intestine of pigs.

\section{REFERENCES}

1. Cheng G, Hao H, Xie S, Wang X, Dai M, Huang L, et al. Antibiotic alternatives: the substitution of antibiotics in animal husbandry? Front Microbiol. 2014;5:217. https://doi.org/10.3389/ fmicb.2014.00217

2. Li J. Current status and prospects for in-feed antibiotics in the different stages of pork production—a review. Asian-Australas J Anim Sci. 2017;30:1667-73. https://doi.org/10.5713/ ajas.17.0418

3. Tollefson L, Karp BE. Human health impact from antimicrobial use in food animals. Med Mal Infect. 2004;34:514-21. https://doi.org/10.1016/j.medmal.2004.07.028

4. Omonijo FA, Ni L, Gong J, Wang Q, Lahaye L, Yang C. Essential oils as alternatives to antibiotics in swine production. Anim Nutr. 2018;4:126-36. https://doi.org/10.1016/ j.aninu.2017.09.001

5. Thacker PA. Alternatives to antibiotics as growth promoters for use in swine production: a review.J Anim Sci Biotechnol. 2013;4:35. https://doi.org/10.1186/2049-1891-4-35

6. Brown K, Uwiera RRE, Kalmokoff ML, Brooks SPJ, Inglis GD. Antimicrobial growth promoter use in livestock: a requirement to understand their modes of action to develop effective alternatives. Int J Antimicrob Agents. 2017;49:12-24. https://doi.org/10.1016/j.ijantimicag.2016.08.006

7. Bengtsson B, Wierup M. Antimicrobial resistance in Scandinavia after a ban of antimicrobial growth promoters. Anim Biotechnol. 2006;17:147-56. https://doi.org/10.1080/104953 90600956920

8. Laine TM, Lyytikäinen T, Yliaho M, Anttila M. Risk factors for post-weaning diarrhoea on piglet producing farms in Finland. Acta Vet Scand. 2008;50:21. https://doi.org/10.1186/17510147-50-21

9. Heo JM, Opapeju FO, Pluske JR, Kim JC, Hampson DJ, Nyachoti CM. Gastrointestinal health and function in weaned pigs: a review of feeding strategies to control post-weaning diarrhoea without using in-feed antimicrobial compounds. J Anim Physiol Anim Nutr. 2013;97:207-37.https://doi.org/10.1111/j.1439-0396.2012.01284.x

10. Sekirov I, Russell SL, Antunes LCM, Finlay BB. Gut microbiota in health and disease. Physiol Rev. 2010;90:859-904. https://doi.org/10.1152/physrev.00045.2009

11. Bengmark S. Gut microbiota, immune development and function. Pharmacol Res. 2013;69:87113. https://doi.org/10.1016/j.phrs.2012.09.002

12. Liu Y, Chen F, Odle J, Lin X, Jacobi SK, Zhu H, et al. Fish oil enhances intestinal integrity and inhibits TLR4 and NOD2 signaling pathways in weaned pigs after LPS challenge.J Nutr. 2012;142:2017-24. https://doi.org/10.3945/jn.112.164947 
13. Issara U, Park S, Park S. Determination of fat accumulation reduction by edible fatty acids and natural waxes in vitro. Food Sci Anim Resour. 2019;39:430-45. https://doi.org/10.5851/kosfa.2019.e38

14. Issara U, Park S, Lee S, Lee J, Park S. Health functionality of dietary oleogel in rats fed highfat diet: a possibility for fat replacement in foods. J Funct Foods. 2020;70:103979. https://doi. org/10.1016/j.jff.2020.103979

15. Park S, Cho S, Hwang O. Effects of Italian Ryegrass (IRG) supplementation on animal performance, gut microbial compositions and odor emission from manure in growing pigs. Agronomy. 2020;10:647. https://doi.org/10.3390/agronomy10050647

16. Lee J, Seo HG, Lee CH. Effects of lotus (Nelumbo nucifera) leaf hot water extracts on the quality and stability of eggs using ultrasonication treatment during storage. Food Sci Anim Resour. 2020;40:1044-54. https://doi.org/10.5851/kosfa.2020.e81

17. Fang J, Yan FY, Kong XF, Ruan Z, Liu ZQ, Huang RL, et al. Dietary supplementation with Acanthopanax senticosus extract enhances gut health in weanling piglets. Livest Sci. 2009;123:268-75. https://doi.org/10.1016/j.livsci.2008.11.020

18. Bird AR, Vuaran M, Crittenden R, Hayakawa T, Playne MJ, Brown IL, et al. Comparative effects of a high-amylose starch and a fructooligosaccharide on fecal bifidobacteria numbers and short-chain fatty acids in pigs fed Bifidobacterium animalis. Dig Dis Sci. 2009;54:947-54. https://doi.org/10.1007/s10620-008-0451-3

19. Kiarie E, Romero LF, Nyachoti CM. The role of added feed enzymes in promoting gut health in swine and poultry. Nutr Res Rev. 2013;26:71-88. https://doi.org/10.1017/ S0954422413000048

20. Azuine MA, Bhide SV. Chemopreventive effect of turmeric against stomach and skin tumors induced by chemical carcinogens in Swiss mice. Nutr Cancer. 1992;17:73-83. https://doi. org/10.1080/01635589209514174

21. Panpatil VV, Tattari S, Kota N, Nimgulkar C, Polasa K. In vitro evaluation on antioxidant and antimicrobial activity of spice extracts of ginger, turmeric and garlic.J Pharmacogn Phytochem. 2013;2:143-8.

22. Chattopadhyay I, Biswas K, Bandyopadhyay U, Banerjee RK. Turmeric and curcumin: biological actions and medicinal applications. Curr Sci. 2004;87:44-53.

23. Dono ND. Turmeric (Curcuma longa Linn.) supplementation as an alternative to antibiotics in poultry diets. Indones Cent Anim Res Dev 2018;23:41-93.

24. Leonel M, Sarmento SBS, Cereda MP. New starches for the food industry: Curcuma longa and Curcuma zedoaria. Carbohydr Polym. 2003;54:385-8.

25. Kuttigounder D, Lingamallu JR, Bhattacharya S. Turmeric powder and starch: selected physical, physicochemical, and microstructural properties. J Food Sci. 2011;76:C1284-91. https:// doi.org/10.1111/j.1750-3841.2011.02403.x

26. Zam W. Gut microbiota as a prospective therapeutic target for curcumin: a review of mutual influence.J Nutr Metab. 2018;2018:1367984. https://doi.org/10.1155/2018/1367984

27. Gupta SC, Patchva S, Aggarwal BB. Therapeutic roles of curcumin: lessons learned from clinical trials. AAPS J. 2013;15:195-218. https://doi.org/10.1208/s12248-012-9432-8

28. Peterson CT, Vaughn AR, Sharma V, Chopra D, Mills PJ, Peterson SN, et al. Effects of turmeric and curcumin dietary supplementation on human gut microbiota: a double-blind, randomized, placebo-controlled pilot study. J Evid Based Integr Med. 2018;23:2515690X18790725. https://doi.org/10.1177/2515690X18790725

29. Ammon HP, Anazodo MI, Safayhi H, Dhawan BN, Srimal RC. Curcumin: a potent inhibitor of leukotriene B4 formation in rat peritoneal polymorphonuclear neutrophils (PMNL). 
1992;58:226. https://doi.org/10.1055/s-2006-961438

30. Negi PS, Jayaprakasha GK, Jagan Mohan Rao L, Sakariah KK. Antibacterial activity of turmeric oil: a byproduct from curcumin manufacture. J Agric Food Chem. 1999;47:4297-300. https://doi.org/10.1021/jf990308d

31. Miquel J, Bernd A, Sempere JM, Díaz-Alperi J, Ramirez A. The curcuma antioxidants: pharmacological effects and prospects for future clinical use. a review. Arch Gerontol Geriatr. 2002;34:37-46. https://doi.org/10.1016/S0167-4943(01)00194-7

32. Maneewan C, Yamauchi KE, Mekbungwan A, Maneewan B, Siri S. Effect of turmeric (Curcuma longa Linnaeus) on growth performance, nutrient digestibility, hematological values, and intestinal histology in nursery pigs.J Swine Health Prod. 2012;20:231-40.

33. Alagbe JO. Growth performance and blood parameters of weaner pigs fed diets supplemented with turmeric powder. Sch J Agric Sci. 2017;7:57-61.

34. National Research Council. Nutrient requirements of swine. Washington, DC: National Academies Press; 2012.

35. Cho S, Hwang O, Park S. Effect of dietary protein levels on composition of odorous compounds and bacterial ecology in pig manure. Asian-Australas J Anim Sci. 2015;28:1362-70. https://doi.org/10.5713/ajas.15.0078

36. Balasubramanian B, Lee SI, Kim IH. Inclusion of dietary multi-species probiotic on growth performance, nutrient digestibility, meat quality traits, faecal microbiota and diarrhoea score in growing-finishing pigs. Ital J Anim Sci. 2018;17:100-6. https://doi.org/10.1080/182805 1X.2017.1340097

37. Kang SJ, Jang CS, Son JM, Hong KW. Comparison of seven commercial TaqMan master mixes and two real-time PCR platforms regarding the rapid detection of porcine DNA. Food Sci Anim Res. 2021;41:85-94. https://doi.org/10.5851/kosfa.2020.e80

38. Platel K, Srinivasan K. Influence of dietary spices and their active principles on pancreatic digestive enzymes in albino rats. Food/Nahrung. 2000;44:42-6. https://doi.org/10.1002/ (SICI)1521-3803(20000101)44:1<42::AID-FOOD42>3.0.CO;2-D

39. Singh PK, Kumar A, Tiwari DP, Kumar A, Palod J. Effect of graded levels of dietary turmeric (Curcuma longa) powder on performance of broiler chicken. Ind J Anim Nutr. 2018;35:42835. http://doi.org/10.5958/2231-6744.2018.00065.8

40. Tubcharoen S, Chomchai S, Saardrak K, Pisarnpong C, Dirokwirat W, Ponglumjeak S. Effects of dietary turmeric meal supplementation on production performance and carcass quality of growing-finishing pigs. In: Proceedings of 45 Kasetsart University Annual Conference; 2007; Bangkok.

41. Etim NN, Offiong EEA, Williams ME, Asuquo LE. Influence of nutrition on blood parameters of pigs. Am J Biol Life Sci. 2014;2:46-52.

42. Shao W, Yu Z, Chiang Y, Yang Y, Chai T, Foltz W, et al. Curcumin prevents high fat diet induced insulin resistance and obesity via attenuating lipogenesis in liver and inflammatory pathway in adipocytes. PLOS ONE. 2012;7:e28784. https://doi.org/10.1371/journal.pone.0028784

43. Porn-anek P, Uriyapongson S, Vichasilp C. Enhanced curcumin solubility by solid dispersion technique reduces fat accumulation in pigs. Food Appl Biosci J. 2017;5:104-14. https://doi. org/10.14456/fabj.2017.9

44. Henningsson Å, Björck I, Nyman M. Short-chain fatty acid formation at fermentation of indigestible carbohydrates. Näringsforskning. 2001;45:165-8. https://doi.org/10.3402/fnr. v45i0.1801

45. Williams BA, Grant LJ, Gidley MJ, Mikkelsen D. Gut fermentation of dietary fibres: physico-chemistry of plant cell walls and implications for health. Int J Mol Sci. 2017;18:2203. 
https://doi.org/10.3390/ijms18102203

46. Park S, Cho E, Chung H, Cho K, Sa S, Balasubramanian B, et al. Digestibility of phosphorous in cereals and co-products for animal feed. Saudi J Biol Sci. 2019;26:373-7. https://doi. org/10.1016/j.sjbs.2018.12.003

47. Zhao J, Bai Y, Tao S, Zhang G, Wang J, Liu L, et al. Fiber-rich foods affected gut bacterial community and short-chain fatty acids production in pig model. J Funct Foods. 2019;57:26674. https://doi.org/10.1016/j.jff.2019.04.009

48. Han KH, Azuma S, Fukushima M. In vitro fermentation of spent turmeric powder with a mixed culture of pig faecal bacteria. Food Funct. 2014;5:2446-52. https://doi.org/10.1039/ C4FO00142G

49. Xun W, Shi L, Zhou H, Hou G, Cao T, Zhao C. Effects of curcumin on growth performance, jejunal mucosal membrane integrity, morphology and immune status in weaned piglets challenged with enterotoxigenic Escherichia coli. Int Immunopharmacol. 2015;27:46-52. https:// doi.org/10.1016/j.intimp.2015.04.038

50. Pluske JR, Thompson MJ, Atwood CS, Bird PH, Williams IH, Hartmann PE. Maintenance of villus height and crypt depth, and enhancement of disaccharide digestion and monosaccharide absorption, in piglets fed on cows' whole milk after weaning. Br J Nutr. 1996;76:409-22. https://doi.org/10.1079/BJN19960046

51. Avanço GB, Ferreira FD, Bomfim NS, de Souza Rodrigues dos Santos PA, Peralta RM, Brugnari T, et al. Curcuma longa L. essential oil composition, antioxidant effect, and effect on Fusarium verticillioides and fumonisin production. Food Control. 2017;73:806-13. https://doi. org/10.1016/j.foodcont.2016.09.032

52. Chen L, Xu Y, Chen X, Fang C, Zhao L, Chen F. The maturing development of gut microbiota in commercial piglets during the weaning transition. Front Microbiol. 2017;8:1688. https:// doi.org/10.3389/fmicb.2017.01688

53. Hu J, Nie Y, Chen J, Zhang Y, Wang Z, Fan Q, et al. Gradual changes of gut microbiota in weaned miniature piglets. Front Microbiol. 2016;7:1727. https://doi.org/10.3389/ fmicb.2016.01727

54. Zhai SS, Ruan D, Zhu YW, Li MC, Ye H, Wang WC, et al. Protective effect of curcumin on ochratoxin a-induced liver oxidative injury in duck is mediated by modulating lipid metabolism and the intestinal microbiota. Poult Sci. 2020;99:1124-34. https://doi.org/10.1016/ j.psj.2019.10.041

55. Lukiw WJ. Bacteroides fragilis lipopolysaccharide and inflammatory signaling in Alzheimer's disease. Front Microbiol. 2016;7:1544. https://doi.org/10.3389/fmicb.2016.01544

56. Zhao Y, Wu J, Li JV, Zhou NY, Tang H, Wang Y. Gut microbiota composition modifies fecal metabolic profiles in mice. J Proteome Res. 2013;12:2987-99. https://doi.org/10.1021/ pr400263n

57. Shen L, Liu L, Ji HF. Regulative effects of curcumin spice administration on gut microbiota and its pharmacological implications. Food Nutr Res. 2017;61:1361780. https://doi.org/10.108 0/16546628.2017.1361780

58. Alauzet C, Marchandin H, Lozniewski A. New insights into Prevotella diversity and medical microbiology. Future Microbiol. 2010;5:1695-718. https://doi.org/10.2217/fmb.10.126

59. Amat S, Lantz H, Munyaka PM, Willing BP. Prevotella in pigs: the positive and negative associations with production and health. Microorganisms. 2020;8:1584. https://doi.org/10.3390/ microorganisms 8101584

60. Kosti D, Dahiya DS, Dalal R, Vinus. Effect of supplementing different levels of turmeric powder on ileal morphology in laying hens. Int J Curr Microbiol Appl Sci. 2018;7:1524-32. https:// 
doi.org/10.20546/ijcmas.2018.707.179

61. Wang J, Ghosh SS, Ghosh S. Curcumin improves intestinal barrier function: modulation of intracellular signaling, and organization of tight junctions. Am J Physiol Cell Physiol. 2017;312:C438-45. https://doi.org/10.1152/ajpcell.00235.2016 